\title{
Chronobiology, chronotherapy and timing of aspirin ingestion
}

\author{
Hannah Cooke-Ariel ${ }^{1 *}$, Sarah K. Wood ${ }^{2}$, Charles H. Hennekens ${ }^{3}$ \\ ${ }^{1}$ Adjunct Professor, Scientific Literacy, Department of Physical Science, Lynn University, USA \\ ${ }^{2}$ Assistant Professor of Biomedical Science and Assistant Dean for, Charles E. Schmidt College of Medicine, Curriculum, Florida Atlantic University, USA \\ ${ }^{3}$ Sir Richard Doll Professor and Senior Academic Advisor to the, Charles E. Schmidt College of Medicine, Florida Atlantic University, USA
}

\section{Chronobiology and Chronopharmacokinetics}

A circadian pattern in activity has been demonstrated for a multitude of physiologic factors affecting cardiovascular function. For example, upon awakening, adrenergic tone is augmented. This heightened sympathetic tone, together with elevations in cortisol release leads to increased vasomotor tone, increased blood pressure, and increases in platelet adhesiveness and aggregation. Activity of thromboxane A2 also follows a circadian pattern of occurrence, with peak activity in early morning hours, or upon awakening. Superimposable upon this circadian activity pattern of physiologic factors is the circadian occurrence of acute ischemic events, arrhythmia, and sudden cardiac death. There is a direct correlation between early morning peak activity of physiologic stressors and acute coronary events.

Chronopharmacokinetic studies have indicated that factors affecting drug disposition also follow a circadian activity pattern. For example Gastric $\mathrm{pH}$ is lowest in early morning hours from 2am to 6am, promoting drug absorption and dispersion. Gastric emptying time is delayed during the hours of sleep, and also with ingestion of food. Pharmacological agents competitively inhibiting the reninangiotensin-aldosterone system are more effective in reducing blood pressure at night, when peak renin-angiotensin-aldosterone activity has been documented to occur. Agents acting to competitively inhibit the adrenergic system are more effective during early morning and daytime hours when heightened sympathetic tone is present.

\section{Chronotherapy}

Chronotherapy refers to the investigation and application of optimal timing of medication, which is based in chronobiology, or time dependent changes. The Physician's Health Study (PHS) was a randomized, double blind placebo controlled $2 \times 2$ factorial trial of aspirin in the primary prevention of cardiovascular disease (CVD) and beta-carotene in the primary prevention of CVD and cancer. The beta carotene component continued to its scheduled termination after 12 years of treatment and follow up and showed no significant evidence of benefit or harm [1]. In contrast, the aspirin component was terminated early based on the unanimous recommendation of the external and multidisciplinary Data and Safety Monitoring Board (DSMB) [2] due primarily to the emergence of a statistically extreme benefit of aspirin of $44 \%$ on the development of a first myocardial infarction (MI) [3]. Subsequently it was shown that about $40 \%$ of first MIs in the PHS occurred between $7 \mathrm{am}$ and $10 \mathrm{am}$, which was significantly higher than the $12.5 \%$ expected under the null hypothesis of no variation by time [4].

\section{Early morning and evening administration of aspirin}

On the basis of chronobiology and chronotherapy clinicians were advised to instruct their patients to take aspirin for long term treatment or primary prevention in the early morning [5]. For patients who are not inclined to take their morning medication upon awakening, however, evening administration may be preferable to late morning or afternoon in order to achieve optimal coverage for the early morning hours. Further, the evening administration of aspirin may, in fact, be optimal in "non-dippers", defined individuals who do not exhibit the normal J-curve of blood pressure. Non-dippers tend to include subpopulations of patients such as diabetics, patients with congestive heart disease, and those with chronic kidney disease. These patients may benefit from evening administration of aspirin [6,7].

The choice of timing as well as whether to prescribe aspirin should be an individual clinical judgement and, thus, left to the discretion of the well informed clinician. The timing should be chosen to promote patient compliance, and to provide protection during the well characterized vulnerable early morning period.

\section{Disclosures}

Drs. Ariel and Wood report no disclosures.

Professor Hennekens reports that he is funded by the Charles E. Schmidt College of Medicine of Florida Atlantic University; serves as an independent scientist in an advisory role to investigators and sponsors as Chair or Member of Data and Safety Monitoring Boards for Amgen, AstraZeneca, Bayer, Bristol Myers-Squibb, British Heart Foundation, Cadila, Canadian Institutes of Health Research, DalCor, Genzyme, Lilly, Regeneron, Sanofi, Sunovion and the Wellcome Foundation. to legal counsel for Pfizer and Takeda, the United States (U.S.) Food and Drug Administration, and Up To Date; and receives royalties for authorship or editorship of 3 textbooks and as coinventor

Correspondence to: Hannah Cooke-Ariel, Adjunct Professor, Scientific Literacy, Department of Physical Science, Lynn University, Boca Raton, Florida 33431, USA, Tel: 561-313-8456, E-mail: HAriel@lynn.edu

Received: April 20, 2015; Accepted: May 20, 2015; Published: May 21, 2015 
on patents for inflammatory markers and CV disease that are held by Brigham and Women's Hospital; and has an investment management relationship with the West-Bacon Group within SunTrust Investment Services, which has discretionary investment authority and does not own any common or preferred stock in any pharmaceutical or medical device company.

\section{References}

1. Hennekens CH, Buring JE, Manson JE, Stampfer M, Rosner B, et al. (1996) Lack of effect of long-term supplementation with beta-carotene on the incidence of malignant neoplasms and cardiovascular disease. N Engl J Med 334: 1145-49. [Crossref]

2. The Steering Committee of the Physicians'Health Study Research Group (1988) Preliminary report: finding from the aspirin component of the ongoing Physicians' Health Study. N Engl J Med 318: 262-64.
3. (1989) Physicians' Health Study:aspirin and primary prevention of coronary heart disease. $N$ Engl J Med 321: 1825-28. [Crossref]

4. Ridker PM, Manson JE, Buring JE, Muller JE, Hennekens CH (1990) Circadian variation of myocardial infarction and the effect of low dose aspirin in a randomized trial of physicians. Circulation 82: 897-902. [Crossref]

5. Recommendations for aspirin for prevention of cardiovascular disease. National Center For Chronic Diseases, Prevention, and Health Promotion, Division for Heart Disease and Stroke Prevention, Sept 2011.

6. Verdeccia P, Schillaci G, Portellati C (1991) Dippers versus non-dippers. J Hypertens Suppl 9: S42-S44. [Crossref]

7. Kitamura T, Onishi K, Dohi K, Okinaka T, Ito M, et al. (2002) Circadian rhythm of blood pressure is transformed from dipper to non-dipper pattern in shift workers with hypertension. J Hum Hypertens 16: 193-197. [Crossref]

Copyright: (O2015 Cooke-Ariel H. This is an open-access article distributed under the terms of the Creative Commons Attribution License, which permits unrestricted use, distribution, and reproduction in any medium, provided the original author and source are credited. 